\title{
A general stability criterion for switched linear systems having stable and unstable subsystems
}

\author{
Jesús San Martín \\ EUITI Universidad Politécnica de Madrid, Ronda de Valencia, 3. 28012 Madrid (Spain) \\ Dep. Física Matemática y de Fluidos, UNED, Po Senda del Rey, 9. 28040 Madrid \\ (Spain) \\ Anthony G. O'Farrell \\ Dept. of Mathematics and Statistics, NUI Maynooth. Co. Kildare, Ireland
}

\begin{abstract}
We report conditions on a switching signal that guarantee that solutions of a switched linear systems converge asymptotically to zero. These conditions are apply to continuous, discrete-time and hybrid switched linear systems, both those having stable subsystems and mixtures of stable and unstable subsystems.
\end{abstract}

Keywords: stability criterion; switched linear system; unstable subsystem

\section{Introduction}

In Science and Engineering, systems are frequently met that consist of a family of subsystems and a switching signal which determines which subsystem is activated each time.

Email addresses: jsm@dfmf.uned.es (Jesús San Martín), admin@maths.nuim.ie (Anthony G. O'Farrell) 
When all the subsystems are linear, one has a switched linear system

$$
\dot{x}(t)=A_{\sigma(t)} x(t)
$$

where $\sigma:[0,+\infty) \rightarrow\{1, \cdots, n\}$ is the switching signal and $A_{i}: \mathbb{R}^{m} \rightarrow$ $\mathbb{R}^{m} \quad(i=1, \cdots, n)$ are matrices that characterise the subsystems. We will assume the condition that there are a finite number of switches in each finite time interval [1, 2].

The great number of areas in which switched linear systems appear makes their study a matter of real concern and great importance [1]. Its theoretical importance [1, 3, 4] derives from its practical importance (power systems, control process, automotive industry, mechanical systems): one needs to understand under what circumstances the system (1) is stable, or what switching signals make the systems stable (see the survey [12 for more details). Many times, time-delay must be taken into account [5, 22] because it plays an important role in many practical systems, such as chemical processes, nuclear reactors, engines, and so on [23, 24].

Liberzon and Morse [1] formulated three basic problems in relation to the stability of switched systems.

"Problem A: Find conditions that guarantee that the switched system is asymptotically stable for any switching signal".

"Problem B: Identify those classes of switching signals for which the switched system is asymptotically stable".

"Problem C: Construct a switching signal that makes the switched system 
asymptotically stable".

The condition of asymptotic stability referred to Problem A, is desirable in practical applications. The theorems that provide solution (or partial solutions) to in Problem $A$ involve conditions that can be restrictive for applications: the existence of Lyapunov functions, symmetric systems, pairwise commutativity of the subsystems, Lie-algebraic conditions, .. [2, 3, 6 , 7]. On the other hand, it is well-known that there exist systems that exhibit instability even though all their subsystems are asymptotically stable [1, 2]. As a result, one sees the necessity of solving Problem $B$ in practice, in order to deal with the applications. Usually, Problem $B$ is studied under the assumption that all the individual subsystems are asymptotically stable [1, 6]. However, for some applications it is convenient to allow subsystems that may be both stable and unstable (for instance, unstable subsystems have to be considered when a failure occurs).

In this paper, we establish conditions on the switching signal of a switched linear system that are sufficient to ensure asymptotic stability. We allow both stable and unstable subsystems in the switched linear system [13]. Furthermore this switched linear system has no restrictive conditions involved, such as the existence of Lyapunov functions, symmetries, pairwise commutativity of the subsystems, Lie-algebraic conditions, etc.

In the following lines we are putting our results in context and indicate which are the novel contributions. As we said above, switched systems with all the subsystems stable can become unstable for certain switching signals. 
The contrary is also true, that switched systems with some of the subsystems unstable can become stable for certain switching signals [14, 6]. From what we have just said it is plain to see that the stability of switched systems depends on both the dynamics of each subsystem and the switching signals.

Intuitively it is easy to imagine a switching signal that maintains stable a switched linear system with all its subsystems stable. It is enough to stay for a long time in one of the subsystems and jump into another with a low frequency. This idea, and the mathematical concept of "low frequency of jumping between subsystems", was developed by Morse and Hespanha with the concepts of dwell time and average dwell time switching [15, 16].

These results about stability were extended in [13] to take into account unstable subsystems, that is switched systems having both stable and unstable subsystems. The underlying idea is very similar to the one just described above. Stay for long times in stable subsystems in such a way that the total activation time of stable subsystems is relatively big compared with that of unstable ones, and furthermore the system should spend long times in every stable subsystems (low frequency of jumping).

Dwell time and average dwell time switching have proved to be very flexible and powerful tools to determine what conditions switching signal should satisfy to guarantee the stability of switched systems. As a result, there is a fruitful line of research since the pioneering ideas to the present day [17, 18, 19, 20]. New approaches using fuzzy time control have also been taken [21]. 
The results shown in this paper also require that stable subsystems control the unstable one, but in a different way. Average dwell time switching requires that the average interval between consecutive switchings must be bigger than a constant, although consecutive switchings can be separated by less than that constant. In contrast to this idea the theorems we show do not need time intervals, we use the number of times that the system switches. We are speaking of a discrete variable in contrast to a continuous one. That is not the only difference. We require that stable subsystems control unstable subsystems pair by pair, as we will explain below. A consequence is that a switched system can spend more time in unstable subsystems than in stable ones and even then the switched system be stable, as is illustrated in example 2.

The paper is organised as follows. First we work on continuous switched linear systems, then on discrete systems. Afterwards we combine these to study hybrid systems. Then we apply our results about problems of type $B$ to the design of switching signals in order to solve problems of type $C$.

\section{Definitions}

The following definitions are necessary for further discussion.

$t_{i_{j}}$ denotes the time during which the system (1) is ruled by matrix $A_{i}$, when this matrix is switched to for the $j$-th time.

$m_{i}(t):[0, \infty) \rightarrow \mathbb{N}$ denotes how many times the matrix $A_{i}$ has been switched up to time $t$. 


\section{Continuous systems}

We first investigate continuous systems, then we will reformulate the results of this section to approach discrete systems.

The next theorem gives us sufficient conditions to get asymptotic stability in a continuous system having both stable and unstable subsystems. It is important to emphasise two things. First, the system will be asymptotically stable even though it has unstable subsystems. Second, we are not using average dwell time switching. This theorem will allow us the stabilisation of a switched linear system by a switching strategy.

Theorem 1. Consider a switched linear system of the form (11) that satisfies:

(i) $m_{i}(t) \rightarrow \infty$ as $t \rightarrow \infty$ for $i=1, \cdots, n$

(ii) $<e^{A_{i}}>=\left[\prod_{i_{j}=1}^{m_{i}}\left\|e^{A_{i} t_{i_{j}}}\right\|\right]^{\frac{1}{m_{i}}} \rightarrow c_{i}<+\infty \quad$ as $\quad m_{i} \rightarrow+\infty$ where

$$
\begin{gathered}
c_{i} \leq 1-\varepsilon_{\beta}, \quad \varepsilon_{\beta}>0, \quad i=1, \cdots, k-1 \\
c_{i} \geq 1, \quad i=k, \cdots, n
\end{gathered}
$$

(iii) $\frac{m_{j}(t)}{m_{\rho}(t)} \rightarrow k_{j, \rho} \geq 1 \quad$ as $t \rightarrow+\infty \quad$ for $\quad \begin{gathered}j=1, \cdots, k-1 \\ \rho=k, \cdots, n\end{gathered}$

(iv) $\prod_{i=1}^{n} c_{i} \leqslant 1-\varepsilon_{\alpha} \quad \varepsilon_{\alpha}>0$

Then each solution $x(t)$ of (1) tends asymptotically to zero.

Significance. 
i) Each matrix $A_{i}$ is used infinitely many times. The process may be random or deterministic. The systems does not stay in any one state as $t \rightarrow \infty$.

ii) The geometric average of the norms of the flows for each matrix $A_{i}$ of (11) is finite. The matrices $A_{j}, j=1, \cdots, k-1$ contract the flow, whereas the matrices $A_{\rho}, \quad \rho=k, \cdots, n$ expand it.

iii) Each contracting matrix dominates each expanding matrix.

iv) The contracting flows dominate the expanding ones.

Proof. After a time $t$, the transfer matrix $A_{i} \quad i=1, \cdots, n$ will have been used $m_{i}(t)$ times. Taking norms it results

$$
\|x(t)\| \leq \prod_{i_{n}=1}^{m_{n}}\left\|e^{A_{n} t_{i_{n}}}\right\| \ldots \prod_{i_{1}=1}^{m_{1}}\left\|e^{A_{1} t_{i_{1}}}\right\|\left\|x_{0}\right\|
$$

it follows from (ii) that

$$
\forall \varepsilon_{i}>0 \quad \exists N_{\varepsilon_{i}} / m_{i}>N_{\varepsilon_{i}} \rightarrow\left|<e^{A_{i}}>-c_{i}\right| \leqslant \varepsilon_{i} \quad i=1, \cdots, n
$$

Let $N_{\varepsilon}=\max _{i=1, \cdots, n}\left\{N_{\varepsilon_{i}}\right\} \quad \varepsilon=\max _{i=1, \cdots, n}\left\{\varepsilon_{i}\right\}$.

If

$$
m_{i}>N_{\varepsilon} \quad i=1, \cdots, n
$$

it follows from (2) that

$$
\|x(t)\| \leq\left(c_{n}+\varepsilon\right)^{m_{n}} \ldots\left(c_{k}+\varepsilon\right)^{m_{k}}\left(c_{k-1}+\varepsilon\right)^{m_{k-1}} \ldots\left(c_{1}+\varepsilon\right)^{m_{1}} \leq
$$




$$
\leq\left(c_{n}+\varepsilon\right)^{M} \ldots\left(c_{k}+\varepsilon\right)^{M}\left(c_{k-1}+\varepsilon\right)^{m} \ldots\left(c_{1}+\varepsilon\right)^{m}\left\|x_{0}\right\|
$$

where $M=\max _{l=k, \cdots, n}\left\{m_{l}\right\}, \quad m=\min _{j=1, \cdots, k-1}\left\{m_{j}\right\}$ and $\varepsilon_{i}$ has been chosen so that $\varepsilon_{i}<\varepsilon_{\beta} \quad i=1, \ldots, n$

It follows from (iii) that

$$
\exists t_{L} / t>t_{L} \rightarrow m \geq M
$$

if

$$
t>t_{L}
$$

it follows from (4) that

$$
\begin{gathered}
\|x(t)\| \leq\left[\left(c_{n}+\varepsilon\right) \ldots\left(c_{1}+\varepsilon\right)\right]^{m}\left\|x_{0}\right\|= \\
=\left(c_{1} \ldots c_{n}+k \varepsilon\right)^{m}\left\|x_{0}\right\| \leq\left(1-\varepsilon_{\alpha}+k \varepsilon\right)^{m}\left\|x_{0}\right\|
\end{gathered}
$$

where (iv) has been used in the last equality.

We choose $\varepsilon<\min \left\{\varepsilon_{\beta}, \frac{\varepsilon_{\alpha}}{k}\right\}$ then $1-\varepsilon_{\alpha}+k \varepsilon<1$

It follows from (i) that

$$
\forall N \quad \exists t_{N} / t>t_{N} \rightarrow m_{i} \geq N \quad i=1, \cdots, n
$$

it is sufficient to take $t>\max \left\{t_{L}, t_{N_{\varepsilon}}\right\}$ so that (3) and (5) are met and consequently (6) with $1-\varepsilon_{\alpha}+k \varepsilon<1$. It follows from (i) that $m \underset{t \rightarrow \infty}{\rightarrow \infty}$. Therefore

$$
\|x(t)\| \leq\left(1-\varepsilon_{\alpha}+k \varepsilon\right)^{m}\left\|x_{0}\right\| \underset{t \rightarrow \infty}{\rightarrow} 0
$$


Presented below is a numerical example in the continuous-time domain.

Example 1. Consider the switched linear system

$$
\begin{gathered}
\dot{x}(t)=A_{\sigma(t)} x(t) \\
\sigma:[0,+\infty) \rightarrow\{1, \cdots, 4\}
\end{gathered}
$$

where switching sequence is generated by random numbers and

$$
\begin{aligned}
& A_{1}=\left(\begin{array}{ll}
-2 & 3 \\
-4 & 5
\end{array}\right) \\
& A_{2}=\left(\begin{array}{rr}
0 & 6 \\
-1 & 5
\end{array}\right) \\
& A_{3}=\left(\begin{array}{rr}
-\frac{29}{4} & \frac{4}{9} \\
-\frac{10}{9} & -\frac{7}{9}
\end{array}\right) \\
& A_{4}=\left(\begin{array}{rr}
-\frac{69}{17} & \frac{2}{17} \\
-\frac{9}{17} & -\frac{50}{17}
\end{array}\right)
\end{aligned}
$$

The subsystems ruled by $A_{1}$ and $A_{2}$ are unstable because their eigenvalues are respectively $\lambda\left(A_{1}\right)=\{1,2\}$ and $\lambda\left(A_{2}\right)=\{2,3\}$. Whereas $A_{3}$ and $A_{4}$, with eigenvalues $\lambda\left(A_{3}\right)=\{-1,-3\}$ and $\lambda\left(A_{4}\right)=\{-3,-4\}$ respectively, determine stable subsystems. 
In order to calculate the evolution of the system (17) the following expressions are needed.

$$
\begin{aligned}
e^{A_{1} t} & =\left(\begin{array}{ll}
-1 & -\frac{3}{4} \\
-1 & -1
\end{array}\right)\left(\begin{array}{ll}
e^{t} & 0 \\
0 & e^{2 t}
\end{array}\right)\left(\begin{array}{rr}
-4 & 3 \\
4 & -4
\end{array}\right) \\
e^{A_{2} t} & =\left(\begin{array}{ll}
3 & 2 \\
1 & 1
\end{array}\right)\left(\begin{array}{ll}
e^{2 t} & 0 \\
0 & e^{3 t}
\end{array}\right)\left(\begin{array}{rr}
1 & -2 \\
-1 & 3
\end{array}\right) \\
e^{A_{3} t} & =\left(\begin{array}{ll}
1 & 2 \\
5 & 1
\end{array}\right)\left(\begin{array}{ll}
e^{-t} & 0 \\
0 & e^{-3 t}
\end{array}\right)\left(\begin{array}{rr}
-\frac{1}{9} & \frac{2}{9} \\
\frac{5}{9} & -\frac{1}{9}
\end{array}\right) \\
e^{A_{4} t} & =\left(\begin{array}{ll}
1 & 2 \\
9 & 1
\end{array}\right)\left(\begin{array}{cc}
e^{-3 t} & 0 \\
0 & e^{-4 t}
\end{array}\right)\left(\begin{array}{rr}
-\frac{1}{17} & \frac{2}{17} \\
\frac{9}{17} & -\frac{1}{17}
\end{array}\right)
\end{aligned}
$$

After 100000 switches, the result is

$$
\begin{aligned}
& \left\langle e^{A_{1}}\right\rangle=\left[\prod_{1_{j}}^{m_{1}}\left\|e^{A_{1} t_{1_{j}}}\right\|_{2}\right]^{\frac{1}{m_{1}}} \underset{m_{1} \rightarrow \infty}{\rightarrow} c_{1}=1.106 \geq 1 \\
& \left\langle e^{A_{2}}\right\rangle=\left[\prod_{2_{j}}^{m_{2}}\left\|e^{A_{2} t_{2_{j}}}\right\|_{2}\right]^{\frac{1}{m_{2}}} \underset{m_{2} \rightarrow \infty}{\rightarrow} c_{2}=1.198 \geq 1 \\
& \left\langle e^{A_{3}}\right\rangle=\left[\prod_{3_{j}}^{m_{3}}\left\|e^{A_{3} t_{3_{j}}}\right\|_{2}\right]^{\frac{1}{m_{3}}} \underset{m_{3} \rightarrow \infty}{\rightarrow} c_{3}=0.985 \leq 1-\varepsilon_{\beta} \quad \varepsilon_{\beta}>0 \\
& \left\langle e^{A_{4}}\right\rangle=\left[\prod_{4_{j}}^{m_{4}}\left\|e^{A_{4} t_{4 j}}\right\|_{2}\right]^{\frac{1}{m_{4}}} \underset{m_{4} \rightarrow \infty}{\rightarrow} c_{4}=0.101 \leq 1-\varepsilon_{\beta} \quad \varepsilon_{\beta}>0
\end{aligned}
$$

with 
$\prod_{i=1}^{4} c_{i}=0.1321-\varepsilon_{\alpha} \quad \varepsilon_{\alpha}>0$

and (see Figure 1)

$\frac{m_{3}}{m_{1}} \underset{t \rightarrow \infty}{\rightarrow} k_{3,1}=1.486$

$\frac{m_{3}}{m_{2}} \underset{t \rightarrow \infty}{\rightarrow} k_{3,2}=1.498$

$\frac{m_{4}}{m_{1}} \underset{t \rightarrow \infty}{\rightarrow} k_{4,1}=1.492$

$\frac{m_{4}}{m_{2}} \underset{t \rightarrow \infty}{\rightarrow} k_{4,2}=1.503$

So theorem conditions from (i) to (iv) are satisfied and the system must asymptotically tend to zero, as it is shown in Figures $2 \mathrm{a}$ and $2 \mathrm{~b}$.

\section{Discrete-time systems}

When time is discrete instead of continuous we have a switched linear discrete-time system, and the system (11) is turned into

$$
x(n+1)=A_{\sigma(t)} x(n)
$$

where $\sigma(t)$ and $A_{\sigma(t)}$ have the same meaning as in system (11).

Discrete-time systems are as useful in engineering as continuous-time sys- 
tems, and theoretical research is also very active. Furthermore, they appear in other areas where continuous systems are not found, as a result of using the transfer matrix method to solve differential equations [8]. Lately, they are becoming more important in the study of structures consisting of stiffened plates (naval architecture, bridge engineering, aircraft design buildings) [9] and spatially periodic structures (satellite antennae, satellite solar panels) [10]. The theorem, stated some lines below, will indicate to a designer how to insert panels (given by $A_{i}$ in (8) ) so that oscillations fade off and do not damage the structure.

The theorem 1 can be reformulated for discrete-time systems in the following way:

Theorem 2. Consider a switched linear discrete-time of the form (88) such that

i) $m_{i}(t) \underset{t \rightarrow \infty}{\rightarrow} \infty \quad i=1, \cdots, n$

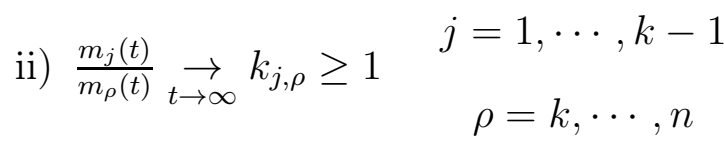

iii) $\left\|A_{j}\right\|<1 \quad j=1, \cdots, k-1$

$$
\left\|A_{\rho}\right\| \geq 1 \quad \rho=k, \cdots, n
$$

iv) $\prod_{i=1}^{n}\left\|A_{i}\right\| \leq 1-\varepsilon_{\alpha} \quad \varepsilon_{\alpha}>0$

Then each solution of (8) tends to 0 as $n \rightarrow+\infty$. 
Proof. After a time $t$ has elapsed, the transfer matrix $A_{i} \quad i=1, \cdots, n$, will have been used $m_{i}(t)$ times. Hence, taking norms we have

$$
\begin{gathered}
\|x(t)\| \leq \prod_{i_{n}=1}^{m_{n}}\left\|A_{n}\right\| \ldots \prod_{i_{1}=1}^{m_{1}}\left\|A_{1}\right\|\left\|x_{0}\right\| \leq \\
\leq\left(\left\|A_{n}\right\| \ldots\left\|A_{k}\right\|\right)^{M}\left(\left\|A_{k-1}\right\| \ldots\left\|A_{1}\right\|\right)^{m}\left\|x_{o}\right\|
\end{gathered}
$$

where $M=\max _{l=k, \cdots, n}\left\{m_{l}\right\}, \quad m=\min _{j=1, \cdots, k-1}\left\{m_{j}\right\}$ and (iii) has been used.

It follows from $(i i)$ that $\exists t_{L} / t>t_{L} \rightarrow m \geq M$.

If

$$
t>t_{L}
$$

it follows from (9) that

$$
\|x(t)\| \leq\left(\left\|A_{n}\right\| \ldots\left\|A_{1}\right\|\right)^{m}\left\|x_{o}\right\| \leq\left(1-\varepsilon_{\alpha}\right)^{m}\left\|x_{o}\right\| \underset{t \rightarrow \infty}{\rightarrow} 0
$$

where (ii) has been used and condition (10) is satisfied when $t \rightarrow \infty$.

If we consider the problem mentioned at the beginning of the section, and imagine a "solar panel" with many sections suffering unstable oscillations then the theorem will indicate the possibility of inserting a panel to extinguish the vibrations.

Giving that the solar panel is a periodical structure, such that the switching to its different components would be ruled by a travelling wave it follows that the switching signal $\sigma(t)$ would be determined by a deterministic expression; hence, the engineer will have to choose the materials in the solar panel so that theorem 2 is satisfied and the travelling wave in it is extinguished. 
A similar argument would allow one to deduce whether a wave would extinguish in a system ruled by Schrödinger or Maxwell equations [11].

Remark 1. It is very easy for engineers to decide whether condition $(i v)$ is satisfied. Given that $\|A\|_{2}=\sqrt{\lambda_{\max }\left(A^{*} A\right)} \quad$ where $\lambda_{\max }\left(A^{*} A\right)=\max$ \{eigenvalues of $\left.A^{*} A\right\}$ it follows that condition $(i v)$ is satisfied if

$$
\left\|A_{n}\right\|_{2} \ldots\left\|A_{1}\right\|_{2}=\prod_{i=1}^{n} \sqrt{\lambda_{\max }\left(A_{i}^{*} A\right)}<1
$$

Below is a numerical example for discrete-time systems.

Example 2. Consider the switched linear system

$$
x(n+1)=A_{\sigma(t)} x(n)
$$

where switching sequence is generated by random numbers and

$$
\begin{aligned}
& A_{1}=\left(\begin{array}{cc}
0 & -\frac{29}{50} \\
\frac{28}{50} & \frac{1}{10}
\end{array}\right) \\
& A_{2}=\left(\begin{array}{cc}
\frac{51}{40} & -\frac{1}{40} \\
-\frac{3}{40} & \frac{49}{40}
\end{array}\right) \\
& A_{3}=\left(\begin{array}{cc}
\frac{57}{50} & \frac{1}{25} \\
\frac{3}{50} & \frac{29}{25}
\end{array}\right) \\
& \left\|A_{1}\right\|_{2} \simeq 0.623<1
\end{aligned}
$$


with eigenvalues $\lambda\left(A_{1}\right)=\left\{\frac{1}{20}+\frac{\sqrt{3223} i}{100}, \frac{1}{20}-\frac{\sqrt{3223} i}{100}\right\}$

$\left\|A_{2}\right\|_{2} \simeq 1.301>1$

with eigenvalues $\lambda\left(A_{2}\right)=\{1.2,1.3\}$

$\left\|A_{3}\right\|_{2} \simeq 1.201>1$

with eigenvalues $\lambda\left(A_{3}\right)=\{1.2,1.1\}$

So, the subsystems ruled by $A_{1}$ is stable, whereas the subsystems ruled by $A_{2}$ and $A_{3}$ are unstable.

After 10000 switches we obtain (see Figure 3)

$$
\begin{aligned}
& \frac{m_{1}(t)}{m_{2}(t)} \underset{t \rightarrow \infty}{\rightarrow} k_{1,2}=1.0158>1 \\
& \frac{m_{1}(t)}{m_{3}(t)} \underset{t \rightarrow \infty}{\rightarrow} k_{1,3}=1.0236>1
\end{aligned}
$$

with

$\left\|A_{1}\right\|_{2}\left\|A_{2}\right\|_{2}\left\|A_{3}\right\|_{2} \simeq 0.973 \leq 1-\varepsilon_{\alpha} \quad \varepsilon_{\alpha}>0$

Given that the conditions of theorem 2 are satisfied the system must asymptotically tend to zero, as it is shown in Figures $4 \mathrm{a}$ and $4 \mathrm{~b}$. 
From (12) and (13) it results that

$$
\frac{m_{2}(t)+m_{3}(t)}{m_{1}(t)} \underset{t \rightarrow \infty}{\rightarrow \sim 2}
$$

(as it can be seen in Figure 3) that is, the system jumps twice as many times into unstable subsystems as it does into stable subsystems. As the switching sequence is generated by random numbers it results, by using the Law of large numbers, that the system spends twice as much time in unstable subsystem as in the stable subsystem. However the system tends asymptotically to zero!

\section{Hybrid system}

When the system has both continuous and discrete subsystems we have a hybrid system.

A linear hybrid system can be described by equations

$$
\begin{gathered}
\dot{x}(t)=A_{\sigma_{1}(t)} x(t) \quad \sigma_{1}(t):[0, \infty) \rightarrow\{1, \cdots, n\} \\
x(t+)=B_{\sigma_{2}(t)} x(t-) \quad \sigma_{2}(t):[0, \infty) \rightarrow\{0,1, \cdots, m\}
\end{gathered}
$$

Here $x(t \pm)$ denote the one-sided limits of $x$ at $t$. The matrix $B_{0}$ is the identity, and the signal $\sigma_{2}(t)$ that controls discrete jumps has $\sigma_{2}(t)=0$ except at a discrete set of times $t$. The system evolves according to the differential equation (14), except when the switching signal $\sigma_{1}(t)$ jumps (also a discrete set of times) or when $\sigma_{2}(t)>0$.

These systems are more and more frequent in industry due to integration 
of continuous and discrete systems. The continuous system might have its origin in the flow or process of a factory or traffic, and the discrete one in the digital control of the diverse steps of the process. Hybrid systems show the same problems formulated by Liberzon y Morse, that we have already mentioned formerly [4]. We can deduce a theorem for these systems that gives the sufficient conditions of stability by using theorems 1 and 2 .

Theorem 3. Suppose a hybrid system given by (14) and (15) is such that the continuous subsystem (14) satisfies the conditions of theorem 1 and the discrete subsystem (15) satisfies the conditions of theorem 2. Then the hybrid system is asymptotically stable.

Proof. It is straight forward. It is enough taking norms of state $x(t)$ after a time $t$, and then to gather separately the norms corresponding to the continuous subsystem and the discrete one. Then proofs of theorem 1 and 2 are respectively repeated for any group.

Remark 2. If one of the subsystems has a bounded solution and the another one tends asymptotically to zero (because it satisfies its respective theorem) then the solution of hybrid system also tends asymptotically to zero. We will return to this remark later.

Example 3. Finally, we consider the hybrid system 14 and 15 formed by the continous one given in example 1 and the discrete system given in example 2. Furthermore the activation of continuous and discrete subsystems is given 
by a switching sequence $\sigma_{3}(t)$ generated by random numbers. As theorem conditions are satisfied the system tends asymptotically to zero as it is shown in Figures $5 \mathrm{a}$ and $5 \mathrm{~b}$.

\section{Aftermaths: stabilisation via the control of the switching signal}

If the switching signal of a switched linear systems is not fixed, but depends on a parameter or can be designed by the engineers, then theorems in this paper allow the design of appropriate feedback laws to make the system stable. Let us show how to do that.

Continuous time.- The condition $(i)$ of theorem 1 shows every subsystem, described by $A_{i}$, must be left before a time $T_{i}$ (see remark 3 below).

Given that

$$
\left\|e^{A_{i} t}\right\| \leq p_{i}(t) e^{\mu_{i} t}
$$

where $\mu_{i}=\max \left\{\operatorname{Re} \lambda_{i} / \lambda_{i}\right.$ eigenvalue of $\left.A_{i}\right\}$ and $p_{i}(t)$ a polynomial of degree the order of $A_{i}$. If we bound $p_{i}(t) \leq k_{i}$ in $\left[0, T_{i}\right]$ it follows that

$$
\left\langle e^{A_{i}}\right\rangle \equiv\left[\prod_{i_{j}=1}^{m_{i}}\left\|e^{A_{i} t_{i_{j}}}\right\|\right]^{\frac{1}{m_{i}}} \leq k_{i} e^{\mu_{i} \overline{t_{i}}}
$$

where

$$
\overline{t_{i}}=\frac{\sum_{i_{j}=1}^{m_{i}} t_{i_{j}}}{m_{i}}
$$

is the average time that system (1) stays in subsystem given by $A_{i}$. 
Therefore

$$
\prod_{i=1}^{n}\left\langle e^{A_{i}}\right\rangle \leq k e^{\sum_{i=1}^{n} \mu_{i} \bar{t}_{i}}
$$

Thus, the time $\overline{t_{i}}$ can be deduced such that theorem 1 is satisfied and asymptotic stability is obtained. It is plain to see that $\sigma(t)$ will not be unique, because we have an average time $\overline{t_{i}}$, that is, engineers can choose any $\sigma(t)$ on the assumption that the average time $\overline{t_{i}}$ satisfies theorem 1 .

It does not matter whether the switched linear system has unstable matrices, the engineer must design the system in such a way that it spends enough time (according to (16)) in stable matrix so that they control the unstable matrices.

Remark 3. The condition of staying a maximum $T_{i}$ in subsystem $A_{i}$ makes a lot of sense from a practical point of view. If the system could be indefinitely in any subsystem then two possibilities would arise:

1. Either the system is ruled by an unstable subsystem and it would be destroyed.

2. Or the system is ruled by a stable subsystem and it would tend asymptotically to zero. Then the result is trivial.

So, the condition is not really a restriction at all.

Discrete time.- In this case, roughly speaking, theorem 2 shows that the system must spend more time evolving under the stable subsystem matrices than under the unstable ones. 
Hybrid system.- The system may have a stable solution because both the continuous and discrete subsystems converge to 0 . Or because the continuous (discrete) subsystem converges to 0 while the discrete (continuous) has a bounded solution (see remark 2).

\section{Conclusions}

We have presented theorems showing that, if a switched linear system has a switching signal such that:

i) The geometric average of the subsystems flow norms is finite;

ii) The geometric averages of stable subsystems dominate the unstable ones;

iii) The stable subsystems control unstable subsystems pair by pair;

then the solution of the system converges asymptotically to zero (even if the system spends more time in unstable subsystems - see example 2). The conditions are for continuous, discrete-time or hybrid systems. These results would allow a practitioner to design the switching signal in order to stabilize the system.

The engineer finds it easier to count how many times the system jumps between different subsystems (see iii) than to calculate an average dwell time switching in order to control the dangerous unstable subsystems. It is easier, and what is more important in practical systems: it is cheaper. Several numerical examples have been presented in order to demonstrate the effectiveness of the theoretical findings. 


\section{References}

[1] Liberzon D, Morse A S. Basic problems in stability and design of switched systems. IEEE Control Systems Magazine 1999; 19(5): 59-70.

[2] Branicky M S. Multiple Lyapunov function and other analysis tools for switched and hybrid systems. IEEE Transactions on Automatic Control 1998; 43(4): 475-482.

[3] Sun Z, Ge S S. Analysis and synthesis of switched linear control systems. Automatica 2005; 41(2): 181-195.

[4] Sun Z, Ge S S. Switched Linear Systems: Control and Design. Springer-Verlag, London, 2005.

[5] Wang D, Wang W, Shi P. Delay-dependent model reduction for continuous-time switched state-delayed systems. International Journal of Adaptive Control and Signal Processing 2011; 25:843-854.

[6] Liberzon D, Hespanha J P, Morse A S. Stability of switched systems: a Lie-algebraic condition. SystemEC Control Letters 1999; 37: $117-122$.

[7] Benzaouia A, El Hajjajim A, Tadeo F. Stabilization of switching Takagi-Sugeno systems by switched Lyapunov function. In- 
ternational Journal of Adaptive Control and Signal Processing 2011; 25: 1039-1049.

[8] Khorasani S, Adibi A. Analytical solution of linear ordinary diferential equations by differential transfer matrix method. Electronic Journal of Differential Equations 2003; 79: 1-18.

[9] Xie W C, Ibrahim A. Buckling mode localization in rib-stiffened plates with misplaced stiffeners-a finite strip approach. Chaos Solitons Er Fractals 2000; 11: 1543-1558.

[10] Xie W C. Vibration mode localization in two-dimensional systems with multiple susbstructural modes. Chaos Solitons \&6 Fractals 2001; 12: 551-570.

[11] Mayer A, Vigneron J P. Accuracy-control techniques applied to stable transfer-matrix computations. Physical Review E 1999; 59: 4659-4666.

[12] Lin H, Antsaklis P J. Stability and Stabilizability of Switched Linear Systems: A Survey of Recent Results. IEEE Transactions on Automatic Control 2009; 54: 308-322

[13] Zhai G, Hu B, Yasuda K, Michel A N. Stability Analysis of Switched Systems with Stable and Unstable Subsystems: An Average Dwell Time Approach. Proceedings of the 2000 American Control Conference 2000; 200-204 
[14] Decarlo R A, Branicky M S, Pettersson S, Lennartson B, Antsaklis P J. Perspectives and results on the stability and stabilizability of hybrid systems. IEEE: Special Issue Hybrid Systems 2000; 88: 1069-1082.

[15] Hespanha J P. Uniform stability of switched linear systems: Extensions of Lasalle's invariance principle. IEEE Trans. Automat. Control 2004; 49 (4): 470-482.

[16] Hespanha J P, Morse A S. Stability of switched systems with average dwell-time. Proc. 38th IEEE Conf. Decision Control, 1999; 2655-2660.

[17] Zhang L, Shi P. Stability, $l_{2}$-Gain and Asynchronous $H_{\infty}$ Control of Discrete-Time Switched Systems with Average Dwell Time. IEEE Trans on Automatic Control. 2009; 54 (9):21932200.

[18] Zhao X, Zhang L, Shi P, Liu M. Stability and stabilization of switched linear systems with mode-dependent average dwell time. IEEE Trans on Automatic Control 2012; 57 (7).

[19] Lian J, Feng Z, Shi P. Observer design for switched recurrent neural networks: an average dwell time approach. IEEE Trans on Neural Networks. 2011; 22 (10): 1547-1556. 
[20] Zhang L, Boukas K, Shi P. $\mu$-Dependent Model Reduction for Uncertain Discrete-Time Switched Linear Systems with Average Dwell Time. Int. J. of Control 2009; 82 (2):378-388.

[21] Wu L, Su X, Shi P and Qiu J. A New Approach to Stability Analysis and Stabilization of Discrete-Time T-S Fuzzy TimeVarying Delay. IEEE Transactions on Systems, Man, and Cybernetics - Part B: Cybernetics 2011;41(1):273-286.

[22] Qiu J, Feng G, Yang J. Delay-dependent non-synchronized robust $\mathscr{H} \infty$ state estimation for discrete-time piecewise linear delay systems. International Journal of Adaptive Control and Signal Processing 2009; 23 (12): 1082-1096.

[23] Guo G. Stability and $H \infty$ performance of multiple-delay systems with successive delay components. International Journal of Adaptive Control and Signal Processing 2010; 24 (8): 643-656.

[24] Orlov Y, Kolmanovsky I V, Gomez O. Adaptive identification of linear time-delay systems: From theory toward application to engine transient fuel identification. International Journal of Adaptive Control and Signal Processing 2009; 23 (2):150-165. 




Figure 1: Temporal evolution of $m_{3} / m_{1}, m_{3} / m_{2}, m_{4} / m_{1}$ and $m_{4} / m_{2}$.

(a)

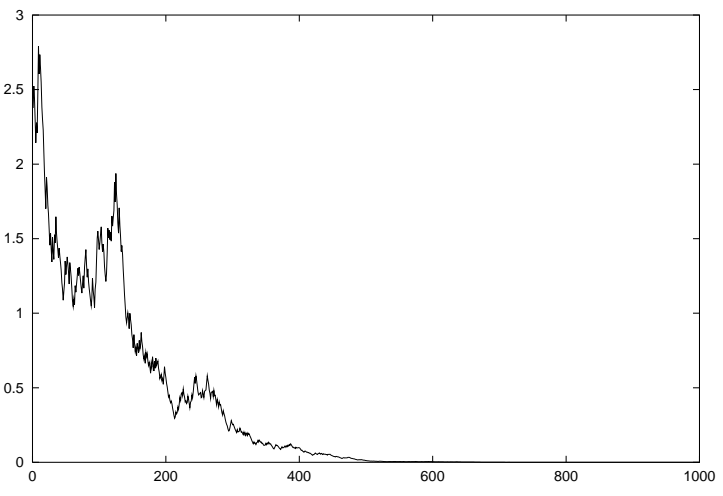

(b)

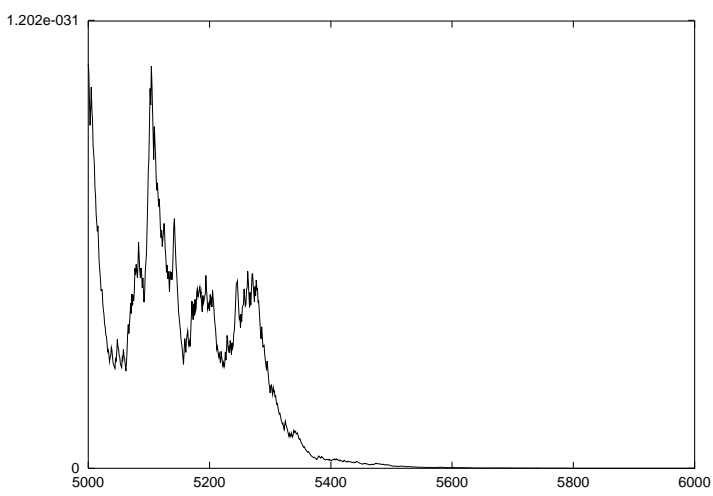

Figure 2: Temporal evolution of the asymptotic convergence in norm to zero of continuous system (7). 


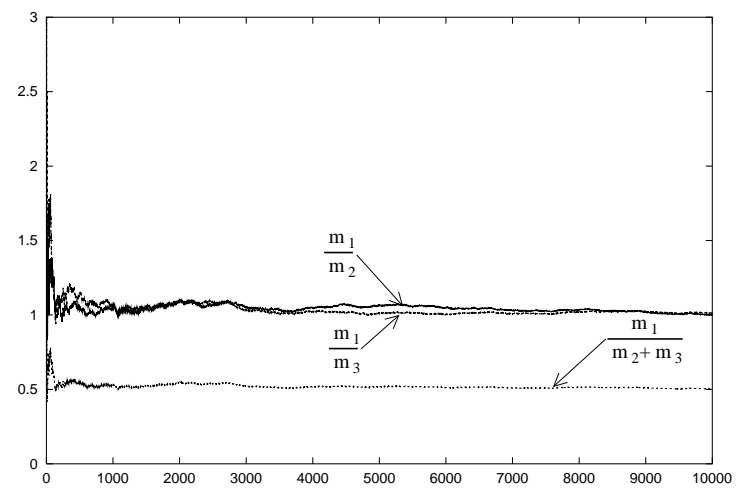

Figure 3: Temporal evolution of $m_{1} / m_{2}, m_{1} / m_{3}$ and $m_{1} /\left(m_{2}+m_{3}\right)$.

(a)

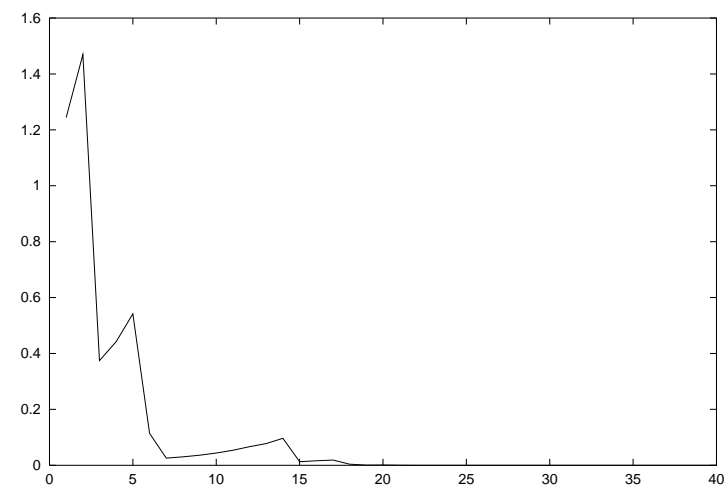

(b)

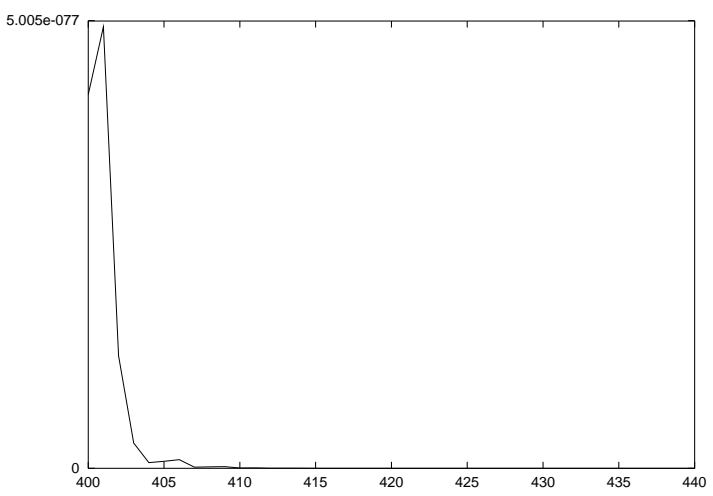

Figure 4: Temporal evolution of the asymptotic convergence in norm to zero of discrete system (11). 


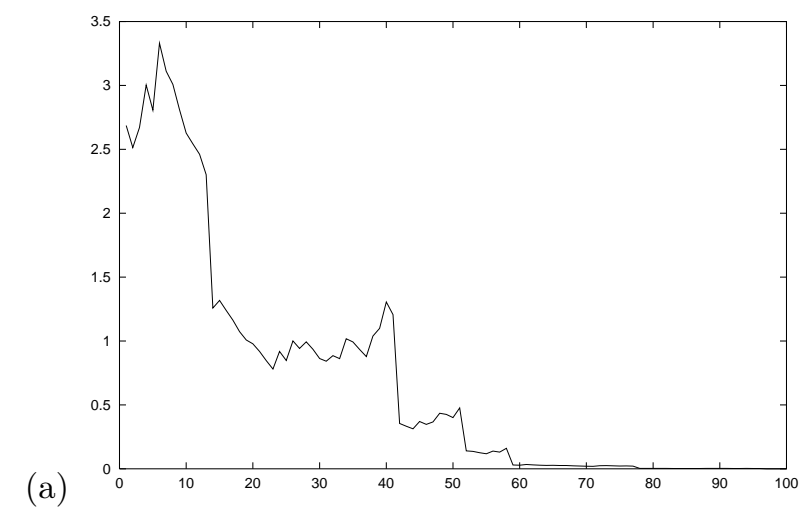

(b)

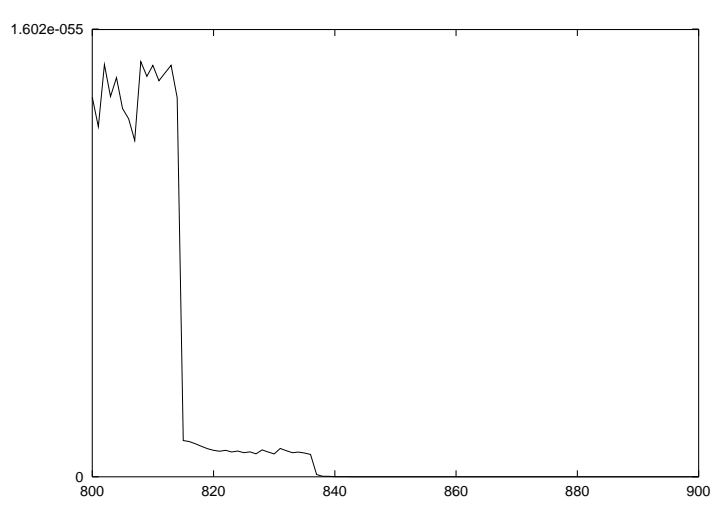

Figure 5: Temporal evolution of the asymptotic convergence in norm to zero of the hybrid system (14)-(15). 